\title{
Darstellung und Reaktivität von zweikernigen Pentamethylcyclopentadienyloxomolybdänsulfiden
}

\author{
Henri Brunner, Heike Kauermann, Joachim Pfauntsch, Joachim Wachter *, \\ Institut für Anorganische Chemie der Universität Regensburg, Universitätsstrasse 31, \\ D.8400 Regensburg (B.R.D.)
}

John H. Enemark und Charles G. Young

The University of Arizona, Department of Chemistry, Tucson, Arizona 85721 (U.S.A.)

(Eingegangen den 26. Januar 1987)

\begin{abstract}
Reaction of $\mathrm{Cp}_{2}^{\star} \mathrm{Mo}_{2} \mathrm{~S}_{4}\left(\mathrm{Cp}^{\star}=\eta^{5}-\mathrm{C}_{5} \mathrm{Me}_{5}\right)$ with oxygen yields two isomers of $\mathrm{Cp}_{2}^{\star} \mathrm{Mo}_{2} \mathrm{OS}_{3}$ and $\mathrm{Cp}_{2}^{\star} \mathrm{Mo}_{2} \mathrm{O}_{2} \mathrm{~S}_{2}$, the constitution of which is determined by means of IR, ${ }^{1} \mathrm{H}$ and ${ }^{95} \mathrm{Mo}$ NMR spectroscopy as well as by X-ray diffraction analysis. The sulfur liberated in this reaction is oxidized and incorporated into an unusually coordinated $\mu, \eta^{1}$-thiosulfate ligand of the compound $\left[\mathrm{Cp}_{2}^{\star} \mathrm{Mo}_{2}\left(\mu, \eta^{2}-\mathrm{S}_{2}\right)(\mu-\mathrm{S})\left(\mu, \eta^{1}\right.\right.$ $\left.\mathrm{S}_{2} \mathrm{O}_{3}\right)$ ]. The reaction of all of these compounds with $(\mathrm{CO})_{5} \mathrm{Cr}(\mathrm{THF})$ and $\left(\mathrm{CH}_{3} \mathrm{CN}\right)_{3} \mathrm{Cr}(\mathrm{CO})_{3}$, respectively, gives clusters either containing a trigonal-bipyramidal $\mathrm{Mo}_{2} \mathrm{CrS}_{2}$ or a cubane-like $\mathrm{Mo}_{2} \mathrm{Cr}_{2} \mathrm{O}_{n} \mathrm{~S}_{4-n}$ skeleton $(n=1,2)$ with five metal-metal bonds. This reaction enables the straightforward incorporation of oxygen atoms into 62-e heterocubane clusters and thus the investigation of ${ }^{95} \mathrm{Mo}$ chemical shifts of mixed oxo/thio-heterocubanes by means of NMR spectroscopy.
\end{abstract}

\section{Zusammenfassung}

Die Reaktion von $\mathrm{Cp}_{2}^{\star} \mathrm{Mo}_{2} \mathrm{~S}_{4}\left(\mathrm{Cp}^{\star}=\eta^{5}-\mathrm{C}_{5} \mathrm{Me}_{5}\right)$ mit Sauerstoff ergibt je zwei Isomere von $\mathrm{Cp}_{2}^{\star} \mathrm{Mo}_{2} \mathrm{OS}_{3}$ und $\mathrm{Cp}_{2}^{\star} \mathrm{Mo}_{2} \mathrm{O}_{2} \mathrm{~S}_{2}$, deren Konstitution mittels IR-, ${ }^{1} \mathrm{H}$ NMR- und ${ }^{95}$ Mo-NMR-Spektroskopie sowie Röntgenstrukturanalyse ermittelt wird. Der bei dieser Reaktion freigewordene Schwefel findet sich in oxidierter Form in einem ungewöhnlich koordinierten $\mu, \eta^{1}$-Thiosulfatliganden der Verbindung $\left[\mathrm{Cp}_{2}^{\star} \mathrm{Mo}_{2}\left(\mu, \eta^{2}-\mathrm{S}_{2}\right)(\mu-\mathrm{S})\left(\mu, \eta^{1}-\mathrm{S}_{2} \mathrm{O}_{3}\right)\right]$ wieder. Die Umsetzung all dieser Komplexe mit $(\mathrm{CO})_{5} \mathrm{Cr}(\mathrm{THF})$ bzw. $\left(\mathrm{CH}_{3} \mathrm{CN}\right)_{3} \mathrm{Cr}(\mathrm{CO})_{3}$ liefert Cluster mit einem trigonal-bipyramidalen $\mathrm{Mo}_{2} \mathrm{CrS}_{2}$ - hzw. einem cubanartigen $\mathrm{Mo}_{2} \mathrm{Cr}_{2} \mathrm{O}_{n} \mathrm{~S}_{4-n}$-Gerüst $(n=1,2)$ mit fünf Metall-Metall-Bindungen. Diese Reaktion ermöglicht den gezielten Einbau von Sauerstoffatomen in 62-e Heterocubancluster and damit die Untersuchung der ${ }^{95}$ Mo-Resonanzen von gemischten Oxo/Thio-Heterocubanen mittels NMRSpektroskopie. 


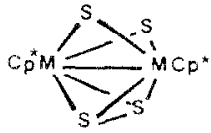

I A :

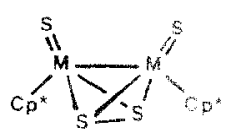

$1 \mathrm{~B}$

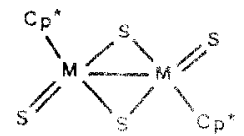

ic:

Schema 1

\section{Einlcitung}

Cyclopentadienyl-gebundene Übergangsmetallsulfide besitzen gegenthber binären Sulfiden den Vorteil der Lösichkeit in organischen Lösungsmitteln. Diese Ergenschaft wird verstärkt durch die Einführung von Alkylsubstituenten in die Cp Reste. wobei gleichzeitig Tendenzen zur Bildung von Metall Schwefelketten unterdrickt werden. Die so gebildeten Liganden, unter denen der Pentamethylowlopentadienylligand $\left(\mathrm{C}^{\star}\right)$ eine Sonderstellung einnimmt, eignen sich besonders fur Stabilisierung von Metallen in formal hohen Oxidationssiufen. Was sich in einer Velfalt ton Komplexen des Typs $C \mathrm{p}_{2}^{\star} \mathrm{M}: \mathrm{S}_{4}$ ausdriicki [1]. Dies gill verstäh fur Cp-gebundene Ubergangsmetalloxide. Die Beispiele $\mathrm{Cp}_{2}^{*} \mathrm{Cr}_{2} \mathrm{O}_{4}[2],\left(\mathrm{C}, \mathrm{H}_{3}, \mathrm{Mo}_{2} \mathrm{O}_{4}, \mathrm{~S}\right], \mathrm{Cp}_{2}^{*} \mathrm{M}_{3} \mathrm{O}_{4}$ [4] und $\mathrm{Cp}_{2}^{\star} \mathrm{Re}_{2} \mathrm{O}_{4}[5]$ stehen für neue, in der Sulfidchemie des enteprechenden Metalls bisher unbekannte Stukturen, so dass eine Verkinplung ven oxdischen mit sulfidischen Strukturelementen in einem Zweikernkomplex reizvoll ersehien. Fir die entsprechenden Versuche wählten wir den für seine vidscitige Reaktivitat belannten Komplex $\mathrm{Cp}_{2}^{\star} \mathrm{Mo}_{2} \mathrm{~S}_{4}[1]$ in Form des sandwichartig aufgebauten lomeren A [6 (Schema 1) aus. unterwarten diesen der erschöpfenden Luftoxidation und untersuchten die Folgeprodukte auf ihre Fähigkeit zur Bildung von Mehrkanclustern. Über einen Teil dieser Arbetten berichteten wir hereits in einer kurmitteilung [7].

\section{Ergebnisse}

Luftoxidation von $\mathrm{Cp}_{2}^{\star} \mathrm{MO}_{2} \mathrm{~S}_{4}(1)$

Rührt man die dunkelblaue Lösung von IA in Toluol an Luft, so lassen sich neben einem wegen seiner mässigen Löslichkeit abfiltrierbaren Produk (II) noch vier weitere Verbindungen (III VI) durch Siulenchromatographie iwolieren (GI. 1).

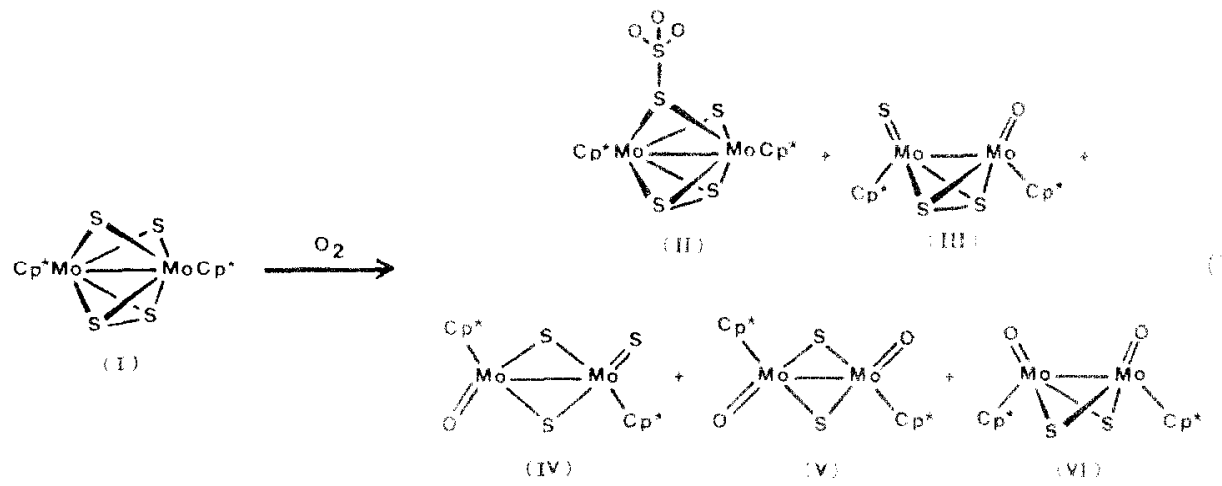

Die Reaktion verläuft optimal bei $50^{\circ} \mathrm{C}$ und einer Dauer von $18 \mathrm{~h}$ es lassen sich dann keine rein schwefelhaltigen Komplexe mehr nachweisen. Elementaranalysen 
und Massenspektren der Verbindungen III-VI lassen auf die Substitution von einem bzw. zwei Schwefelliganden aus IA durch Sauerstoff schliessen. Die Zusammensetzung von II konnte erst mit Hilfe einer Röntgenstrukturanalyse bestimmt werden. II enthält demnach eine an einen $\mu$-S-Liganden addierte $\mathrm{SO}_{3}$-Einheit, während das restliche Molekülskelett mit IA nahezu identisch ist [7]. Auch die durch thermische Isomerisierung aus IA erhältlichen Komplexe IB und IC [6] reagieren an Luft unter Bildung von Oxoverbindungen: ausgehend von IB bilden sich die Komplexe III-VI, während IC lediglich zwei Isomere der Zusammensetzung $\mathrm{Cp}_{2}^{\star} \mathrm{Mo}_{2} \mathrm{O}_{2} \mathrm{~S}_{2}$ liefert. Eines dieser Isomeren besitzt laut Röntgenstrukturanalyse einen planaren $\mathrm{Mo}_{2} \mathrm{~S}_{2}$-Kern mit anti-ständigen terminalen Sauerstoffatomen. Eine Korrelation mit spektroskopischen Daten erfolgte nicht [8].

Die nachfolgenden strukturellen Betrachtungen stützen sich in erster Linie auf IR-, ${ }^{1} \mathrm{H}$ - und ${ }^{95}$ Mo-NMR-Spektren. Einkristalle konnten lediglich von $\mathrm{V}$ erhalten werden. Alle IR-Spektren von III-VI enthalten je eine starke, für eine terminale $\mathrm{Mo}=\mathrm{O}-$ Gruppierung charakteristische Absorption um $900 \mathrm{~cm}^{-1}$ (Tab. 1). Diese wird in III und IV durch eine terminale $\nu(\mathrm{Mo}=\mathrm{S})$-Frequenz ergänzt. Mit Hilfe der ${ }^{1}$ H-NMR-Spektren lassen sich die Verbindungen in Komplexe mit symmetrischem (II, V, VI) bzw. nicht symmetrischem Aufbau (III, IV) einteilen. Die ${ }^{95} \mathrm{Mo}-\mathrm{NMR}-$ Spektren bieten den Vorteil, dass die chemischen Verschiebungen empfindlich auf Änderungen im Sauerstoff- und Schwefelgehalt innerhalb der Koordinationssphäre eines Mo-Atoms reagicren [9]. In Übcreinstimmung hiermit zeigen Komplexe mit ähnlichem Gerüst wie I und II ähnliche $\delta$-Werte (440 [9] bzw. 333 ppm). Die grossen Unterschiede in den chemischen Verschiebungen der Komplexe III and IV von bis zu $753 \mathrm{ppm}$ kommen durch den im Vergleich zu Sauerstoffliganden stark entschirmenden Einfluss der terminalen $\mathrm{S}^{2-}$-Liganden zustande. Ähnliche Differenzen sind auch für andere $\mathrm{Mo}^{\mathrm{V}}$-Komplexe mit dem $\left[\mathrm{Mo}_{2} \mathrm{O}_{2}(\mu-\mathrm{S})_{2}\right]^{2+}$-Kern beobachtet worden [10]. Allerdings sind die Mo-Zentren der Komplexe II-VI besser abgeschirmt als in früher studierten $\mathrm{Mo}^{\mathrm{V}}$-Analoga [11].

Für $\mathrm{Mo}^{\mathrm{V}}$-Komplexe mit dem $\left[\mathrm{Mo}_{2} \mathrm{X}_{4}\right]^{2+}$-Kern $(\mathrm{X}=\mathrm{O}, \mathrm{S})$ sind die Strukturtypen A-D (Schema 2) denkbar. Eine Typ A gemässe, sandwichartige Anordnung scheidet

Tahelle 1

IR-Spektren der Komplexe II-X $\left(\mathrm{KBr}, \mathrm{cm}^{-1}\right)$

\begin{tabular}{llllll}
\hline & $\nu(\mathrm{CO})$ & $\nu(\mathrm{SO})$ & $\nu(\mathrm{Mo}=\mathrm{O})$ & $\nu(\mathrm{Mo}=\mathrm{S})$ & $\nu(\mathrm{S}-\mathrm{S})$ \\
\hline II & - & $1246 \mathrm{vs}, 1207 \mathrm{vs}, 1217 \mathrm{sh}$, & - & - & $515 \mathrm{w}$ \\
& & $1010 \mathrm{vs}, 600 \mathrm{vs}$ & & \\
III & - & - & $900 \mathrm{vs}$ & $483 \mathrm{~s}$ & $492 \mathrm{sh}$ \\
IV & - & - & $898 \mathrm{vs}$ & $490 \mathrm{~s}$ & - \\
V & - & - & $897 \mathrm{vs}$ & - & - \\
VI & - & - & $912 \mathrm{vs}$ & - & - \\
VII & $2003 \mathrm{vs}, 1958 \mathrm{vs}, 1944 \mathrm{sh}$, & - & - & - & - \\
& $1891 \mathrm{vs}{ }^{a}$ & - & - & - \\
VIII & $1936 \mathrm{vs}, 1879 \mathrm{~s}, 1823 \mathrm{~s}^{a}$ & - & - & - & - \\
IX & $2001 \mathrm{vs}, 1970 \mathrm{~m}, 1933 \mathrm{vs}$, & - & - & - & - \\
& $1833 \mathrm{~s}^{b}$ & & & & \\
X & $2005 \mathrm{vs}, 1972 \mathrm{~m}, 1925 \mathrm{vs}$, & - & & & - \\
\end{tabular}

${ }^{a}$ In $\mathrm{CH}_{2} \mathrm{Cl}_{2 \cdot}{ }^{b}$ In Toluol. 
Tabelle 2

NMR-spektroskopische Daten der Komplexe 11 . X

\begin{tabular}{|c|c|c|}
\hline & $\begin{array}{l}\delta\left(^{1} \mathrm{H}\right)^{1} \\
(\mathrm{CH}, \text { Oruppe })\end{array}$ & $\delta 0^{45} \mathrm{M}(1)$ \\
\hline II & 2.39 & $333(140)$ \\
\hline III & 205.198 & $599(100) .541100)$ \\
\hline IV & 2.08 .1 .98 & $440(90-261+20$ \\
\hline $\mathrm{v}$ & 208 & \\
\hline V! & 2.00 & $-9.9(10)$ \\
\hline VII & 1.87 & \\
\hline VIII & 1.87 & $-399(140)$ \\
\hline IX & $1.86,1.79$ & $-265(20,-47 ! 20)$ \\
\hline$x$ & 181 & $-272(20)$ \\
\hline
\end{tabular}

" $\mathrm{CDCl}_{3}$-Lösung. i-TMS, alle Signale Singuletts; Gerät Varian FM 360-L " CH, Cl ,Lönung: in Klammern: Linienbreite in $\mathrm{Hz}$; Gerät Bruker WM 250. $\mathrm{Na}_{2}\left[\mathrm{MoO}_{4}\right]$ extern.

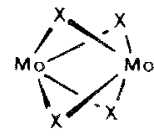

(A)

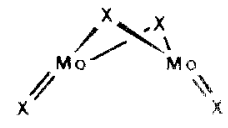

(B)

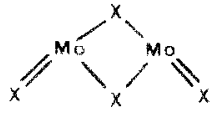

i६!

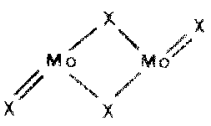

(D)

Schema 2

zumindest für $\mathrm{Cp}_{2} \mathrm{Mo}_{2} \mathrm{~S}_{4}$ aus theoretischen Gründen aus [12]. Fur die Typen B $(\operatorname{syn}), \quad \mathbf{C}$ (closed) und D (anti) wurde eine Abnahme der relativen Stabilitat $\mathbf{B}>\mathbf{C}>\mathbf{D}$ aus elektronischen Grinden vorhergesagt [13]. In Ubereinstimmung hiermit wurden bisher überwiegend Komplexe som sp-Typ gefunden. der closedTyp konnte dagegen bisher noch nicht realisiert werden [13,14]. Eine Destabilisierung der syn-Formen aus sterischen Gründen soll die Einführung des Cyclopentadienylliganden bewirken 144. Tatsächlich gehoren die bishot hekannten Strukturbeispiele aus der Serie $\mathrm{Cp}_{2} \mathrm{Mo}_{2} \mathrm{O}_{2} \mathrm{~S}_{4,},\left(\mathrm{Cp}=\mathrm{C}_{3} \mathrm{H}_{3}, \mathrm{Me}_{5}, n=0.2\right)$ alle Typ $\mathbf{D}$ an [8.15]. Ausnahmen bilden her zwei Strukturen des Typs B fur CP. $\mathrm{MoO}_{4}$ $[3,4]$. Noch keine strukturellen Erfahrungswerte begen fur Komplex mit in $=1.3$ vor.

Die endgültige Zuordnung von $V$ zum Strukturtyp $D$ erfolgte mittels Röntgenstrukturanalyse [16]. Da deren Ergebnis sich mit einer bereits früher ermittelten Struktur vollkommen deckt [8], weshalb hier auf eine Wiedergabe der Strukturparameter verzichtet wird. wird de Zuordnung der orangen Verhindung Vl zum Typ B sehr wahrscheinlich. III und IV sind die ersten nicht smmetrisoly atugebuten Verbindungen aus der Serie $\mathrm{Cp}_{2} \mathrm{Mo}_{2} \mathrm{O}_{n} \mathrm{~S}_{4, n}(n=1-4)$. Witrend III den cinzigen $M 0^{N}$-Komplex in dieser Reihe repräsentiert, wird IV aufgrund seiner Farbanalogie zu den ebenfalls braunen verbindungen antim $\mathrm{Cp} M \circ,(\mu-5), 5$. [8] und $\checkmark$ dem Strukturtyp D zugeordnet.

Die Komplexe II-VI als Bausteine bei der Clustersynthese

Der Versuch, die Komplexe II-VI mit $(\mathrm{CO})_{5} \mathrm{Cr}$ (THF) umzusetzen, brachte nur von II ausgehend ein brauchbares Ergebnis. Als Hauptprodukt bildete sich in $20 \%$ 
Ausbeute laut FD-Massenspektrum und Elementaranalysen der Dreikerncluster VIII neben geringfügigen Mengen an I. Überraschenderweise enthält das IRSpektrum von VIII nur mehr Absorptionen im Bereich terminaler CO-Liganden (Tab. 1), das Fehlen der in II vorhandenen starken $\nu(\mathrm{S}=\mathrm{O})$-Frequenzen deutet auf den Verlust der $\mathrm{SO}_{3}$-Einheit. Auch die Rückbildung von $\mathrm{I}$ ist ein Indiz für die Labilität des Thiosulfatliganden in II. Diese Beobachtung findet ihr strukturelles Pendant in einem sehr langen S-S-Abstand (2.168(2) $\AA$ ) [7]. VIII lässt sich auch aus I und $(\mathrm{CO})_{5} \mathrm{Cr}(\mathrm{THF})$ darstellen, begleitet von dem vermutlich cubanartig aufgebauten Komplex VII, der allerdings nicht analysenrein erhalten werden konnte. Der Aufbau von VII und VIII geht aus einem Vergleich mit den isoelektronischen, strukturell gesicherten Clustern $\mathrm{Cp}_{2}^{\star} \mathrm{Mo}_{2} \mathrm{~S}_{4} \mathrm{Fe}(\mathrm{CO})_{2}$ (XI) und $\mathrm{Cp}_{2}^{\star} \mathrm{Mo}_{2} \mathrm{~S}_{4} \mathrm{Fe}_{2}(\mathrm{CO})_{4}$ (XII) hervor [17]. Auch der zu VII homologe Mo-Cluster konnte bereits dargestellt werden [17].

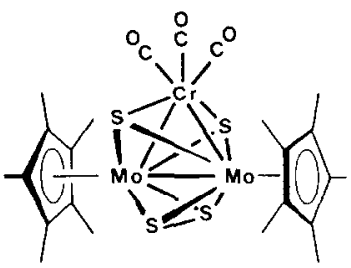

( VIII)

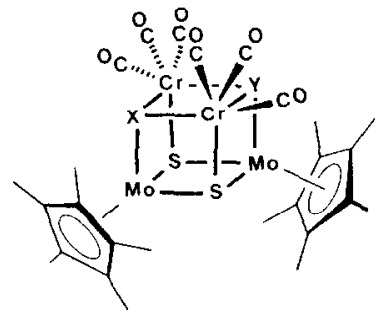

$$
\begin{gathered}
(\text { VII, } X=Y=S ; \\
\text { IX, } X=0, Y=S ; \\
X, X=Y=0)
\end{gathered}
$$

III und $\mathrm{V}$ lassen sich mit $\left(\mathrm{CH}_{3} \mathrm{CN}\right)_{3} \mathrm{Cr}(\mathrm{CO})_{3}$ in guten Ausbeuten in die Cluster IX und X überführen, zu VIII analoge Dreikerncluster wurden nicht gefunden. Wie bereits VII lassen sich IX und X als 62-e Cluster beschreiben, deren Kern aus einem leicht verzerrten $\mathrm{Mo}_{2} \mathrm{Cr}_{2}$-Tetraeder mit fünf Metall-Metall-Bindungen besteht (keine Bindung ist zwischen den beiden $\mathrm{Cr}$-Atomen zu erwarten). Die Addition der 12-e Fragmente $\mathrm{Mo}(\mathrm{CO})_{3}, \mathrm{Fe}(\mathrm{CO})_{2}$ und $\mathrm{Ni}(\mathrm{CO})$ an die potentiellen 38-e Vorstufen IA-C ergibt unter Umbildung des Schwefelligandengerüsts, an der auch Redoxvorgänge beteiligt sind (z.B. $\mathrm{S}_{2}{ }^{2-}+2 e \rightleftharpoons 2 \mu_{3}-\mathrm{S}^{2-}$ ) die strukturell gesicherten $62-e$ Heterocubane $\mathrm{Cp}_{2}^{\star} \mathrm{Mo}_{4}(\mathrm{CO})_{6} \mathrm{~S}_{4}$ [17], XII [17] und $\mathrm{Cp}_{2}^{\star} \mathrm{Mo}_{2} \mathrm{~S}_{4} \mathrm{Ni}_{2}(\mathrm{CO})_{2}$ [18]. Die Konstruktion von gemischten $\mathrm{Oxo} / \mathrm{Thio}-\mathrm{Heterocubanen}$ folgt somit dem gleichen Prinzip und liefert eine Synthesemethode auch zum gezielten Einbau von Sauerstoffatomen in derartige Cluster.

Bedingt durch ihren strukturellen Aufbau befinden sich die Cluster VII-X in Nachbarschaft zu katalytisch oder biologisch relevanten Molekülen, die sich ${ }^{95} \mathrm{Mo}$ NMR-spektroskopisch untersuchen lassen. Wie aus Tab. 2 hervorgeht, erfahren die Mo-Zentren von VII-X im Vergleich zu den Vorstufen II-VI durch die Clusterbildung eine zusätzliche Abschirmung. Hierfür können wohl die Gegenwart von $\mu_{3}-\mathrm{O}-$ bzw. $\mu_{3}$-S-Liganden, die auf +3 reduzierte Formalladung der Mo-Atome und deren direkte Verknüpfung mit carbonylreichen Metallzentren verantwortlich gemacht werden. Ein Vergleich der chemischen Verschiebungen von VIII and XI ( -133 ppm [9]) zeigt für den trigonal-bipyramidalen Clustertypus mit side-on koordiniertem $\mathrm{S}_{2}$-Liganden $\delta$-Werte in einem vergleichbaren Bereich.

Ähnliche, wenngleich höhere $\delta$-Werte als in VIII, sind für IX $(-471 \mathrm{ppm})$ und XII ( $-506 \mathrm{ppm}[9])$ charakteristisch. Der $\delta$-Wert von -272 für X legt somit nahe, 
dass die Resonanz bei $-265 \mathrm{ppm}$ im Spektrum von IX einem $\mu_{3}$-O-Mo-Zentrum zuzuordnen ist. Diese Zuordnung steht allerdings im Gegensatz. zu allen bisher beobachteten Abschirmtrends für Mo-Komplexe mit terminalen oder verbrücken. den Oxo- bew. Sulfidoliganden $[10,11,19]$,

\section{Diskussion}

Schwefelhaltige Übergangsmetallkomplexe reagieren an Luft in der Regel unter Oxidation sowohl verbrückter Mono- als auch verbrückter und endständiger Disulfidliganden, was auch für Natriumperiodat bzw. 3-Chlorperbenzoesäure als Sauerstoffüberträger gilt [20]. Als ersses Beispiel einer Oxygenierung won Metall. sulfiden am Metallzentrum wurde die Luftoxidation eines Cp Wi-Sulfids heshrieben [21]. Auch die Komplexe III - VI tragen die Merkmale einer Reaktion am Metallzentrum. während man sich 11 durch Addition des Elektrophils $\mathrm{SO}_{3}$ an einen $\mu$-S-Liganden von I entstanden denken kann. Eine Erklärung fï dieses Verhalten bietet die Fähigkeit von IA zur Offnung seiner Monosulfubrucken in terminale Sulfidliganden. wobei die Formalladung +4 des Metalls erhahen bleibt [6]. Erst der Bruch der Disulfidhrücke mit nachfolgender Finebnung der Mo. S...Finheit kann formal durch die intramolekulare Redoxreaktion $S_{2}^{2}+2 \mathrm{Mo}^{12}=2 S^{2}+2 \mathrm{Ml}$ ausgedrückt werden. Da in den Oxo-Produkten III- VI nut terminale Sauerstoflliganden gefunden werden, liegt die Vermutung nahe, dass die Reaktwn von $1 \mathrm{~A}$ mi Luftsauerstoff als reine Substitution terminaler Schwefelliganden mit vorgelagerter Isomerisierung aufgefasst werden kann. In Übereinstimmung hiermit reagieren IB und $\mathrm{IC}$ ebenfalls mit $\mathrm{O}_{2}$ ab. Auch mildere Reaktionsbedingungen durch den Einsatz von 3-Chlor-perbenzoesäure bei Raumtemperatur andern wichts an der für de Reaktion von I mit O, charakteristischen Produktverteilung.

\section{Experimenteller Teil}

Alle Arbeiten wurden, soweit nicht anders erwähnt, unter Luftausschluss und unter Verwendung von trockenen, $\mathrm{N}_{2}$-gesättigten Lösungsmitteln durchgeführt. Die Aufnahme der 'H-NMR-Spektren erfolgte an Varian EM 360-L hzw. Bruker WM 250 Geräten. Die ${ }^{95}$ Mo-NMR-Spektren wurden an eincm Bruker WM 250 Cierät gegen $\mathrm{Na}_{2} \mathrm{MoO}_{4}$ als externer Referenz gemessen [9]. Die Elementaranalysen wurden in den Mikroanalytischen Laboratorien der Fakultä Chemie-Pharmaze der Universität Regensburg (C.H) und den Analytischen Laboratorien Prof. Dipl. Ing. Dr. H. Malissa \& Reuter, D-5250 Engelskirchen (S,O) durchgefuhrt. Sic finden sich mi den Ausbeuten und weiteren Eigenschaften in Tab. 3 zusammengefass

\section{Luftoxidation von $\left(\mathrm{C}_{5} \mathrm{Me}_{5}\right)_{2} \mathrm{MO}_{2} \mathrm{~S}_{4}(\mathrm{IA})$}

Eine Lösung von $1.33 \mathrm{~g}$ (2.25 mmol) IA in $100 \mathrm{ml}$ Toluol wird $18 \mathrm{~h}$ bei $50^{\circ} \mathrm{C}$ an Luft gerührt. Der hierbei abgeschiedene rotviolette Niederschlag wird abfiltriert (Aufarbeitung des Filtrats $s, u$.) und mit $20 \mathrm{ml}$ Et, $O$ gewaschen. Die weitere

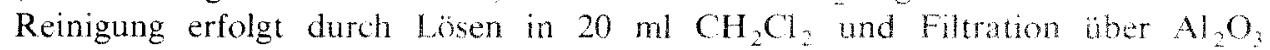
(Aktivität II/III), wobei mit $\mathrm{CH}_{2} \mathrm{Cl}_{2}$ zunächst enthaltene verunreinigungen ausgewaschen werden. Sodann eluiert man mit Aceton II als rotviolettes Produkt. das aus Aceton $/ \mathrm{CH}_{2} \mathrm{Cl}, 5 / 8$ bei $\cdots 25^{\circ} \mathrm{C}$ umkristallisiert watden kann 
Tabelle 3

Ausgewählte Eigenschaften, Ausbeuten und Analysenwerte der Komplexe II-X

\begin{tabular}{|c|c|c|c|c|c|c|c|}
\hline \multirow[t]{2}{*}{ Summenformel } & \multirow[t]{2}{*}{ Farbe } & \multirow{2}{*}{$\begin{array}{l}\text { Ausbeute } \\
(\%)\end{array}$} & \multirow{2}{*}{$\begin{array}{l}\text { Molgew. } \\
\text { (Gef. (ber.)) }\end{array}$} & \multicolumn{4}{|c|}{ Analysen (Gef. (ber.) (\%)) } \\
\hline & & & & $\overline{\mathrm{C}}$ & $\mathrm{H}$ & $S$ & $\mathrm{O}$ \\
\hline $\begin{array}{l}\mathrm{C}_{20} \mathrm{H}_{30} \mathrm{Mo}_{2} \mathrm{O}_{3} \mathrm{~S}_{5} \\
\text { (II) }\end{array}$ & rotviolett & 17 & $\begin{array}{r}649 a \\
(670.6)\end{array}$ & $\begin{array}{c}35.99 \\
(35.82)\end{array}$ & $\begin{array}{c}4.84 \\
(4.51)\end{array}$ & $\begin{array}{c}25.12 \\
(23.90)\end{array}$ & $\begin{array}{c}8.07 \\
(7.16)\end{array}$ \\
\hline $\begin{array}{l}\mathrm{C}_{20} \mathrm{H}_{30} \mathrm{Mo}_{2} \mathrm{OS}_{3} \\
\text { (III) }\end{array}$ & grün & 12 & $\begin{array}{r}566^{b} \\
(574.5)\end{array}$ & $\begin{array}{c}42.10 \\
(41.81)\end{array}$ & $\begin{array}{c}5.05 \\
(5.26)\end{array}$ & $\begin{array}{c}16.68 \\
(16.74)\end{array}$ & \\
\hline $\begin{array}{l}\mathrm{C}_{20} \mathrm{H}_{30} \mathrm{Mo}_{2} \mathrm{OS}_{3} \\
\text { (IV) }\end{array}$ & braun & 5.3 & $\begin{array}{l}566^{b} \\
(574.5)\end{array}$ & $\begin{array}{c}41.60 \\
(41.81)\end{array}$ & $\begin{array}{c}5.75 \\
(5.26)\end{array}$ & $\begin{array}{l}17.38 \\
(16.74)\end{array}$ & \\
\hline $\begin{array}{l}\mathrm{C}_{20} \mathrm{H}_{30} \mathrm{Mo}_{2} \mathrm{O}_{2} \mathrm{~S}_{2} \\
\text { (V) }\end{array}$ & braun & 6.7 & $\begin{array}{l}550^{b} \\
(558.5)\end{array}$ & $\begin{array}{c}42.52 \\
(43.01)\end{array}$ & $\begin{array}{c}5.31 \\
(5.42)\end{array}$ & & \\
\hline $\begin{array}{l}\mathrm{C}_{20} \mathrm{H}_{30} \mathrm{Mo}_{2} \mathrm{O}_{2} \mathrm{~S}_{2} \\
\text { (VI) }\end{array}$ & orange & 7.3 & $\begin{array}{c}550^{b} \\
(558.5)\end{array}$ & $\begin{array}{c}42.90 \\
(43.01)\end{array}$ & $\begin{array}{c}5.39 \\
(5.42)\end{array}$ & & \\
\hline $\begin{array}{l}\mathrm{C}_{26} \mathrm{H}_{30} \mathrm{Cr}_{2} \mathrm{Mo}_{2} \mathrm{O}_{6} \mathrm{~S}_{4} \\
\text { (VII) }\end{array}$ & grün & 9.8 & $\overline{-}(862.6)$ & & & & \\
\hline $\begin{array}{l}\mathrm{C}_{23} \mathrm{H}_{30} \mathrm{CrMo}_{2} \mathrm{O}_{3} \mathrm{~S}_{4} \\
\text { (VIII) }\end{array}$ & rotviolett & 15 & $\begin{array}{l}720^{c} \\
(726.6)\end{array}$ & $\begin{array}{c}38.08 \\
(38.01)\end{array}$ & $\begin{array}{c}4.55 \\
(4.16)\end{array}$ & $\begin{array}{c}17.60 \\
(17.65)\end{array}$ & \\
\hline $\begin{array}{l}\mathrm{C}_{26} \mathrm{H}_{30} \mathrm{Cr}_{2} \mathrm{Mo}_{2} \mathrm{O}_{7} \mathrm{~S}_{3} \\
\quad(\mathrm{IX})\end{array}$ & grün & 46 & $\begin{array}{r}835^{a} \\
(846.6)\end{array}$ & $\begin{array}{l}36.93 \\
(36.89)\end{array}$ & $\begin{array}{c}3.64 \\
(3.57)\end{array}$ & $\begin{array}{c}10.90 \\
(11.36)\end{array}$ & $\begin{array}{r}13.32 \\
(13.23)\end{array}$ \\
\hline $\begin{array}{l}\mathrm{C}_{26} \mathrm{H}_{30} \mathrm{Cr}_{2} \mathrm{Mo}_{2} \mathrm{O}_{8} \mathrm{~S}_{2} \\
\quad(\mathrm{X})\end{array}$ & braun & 32 & $\begin{array}{r}827^{a} \\
(830.5)\end{array}$ & $\begin{array}{c}37.57 \\
(37.60)\end{array}$ & $\begin{array}{c}3.71 \\
(3.64)\end{array}$ & $\begin{array}{c}7.13 \\
(7.72)\end{array}$ & \\
\hline
\end{tabular}

${ }^{a}$ Osmometrisch in $\mathrm{CHCl}_{3}{ }^{b} 70$ eV-Massenspektrum (Gerät Varian $\mathrm{CH}$ ) bzgl. ${ }^{92}$ Mo. ${ }^{c}$ FD-Massenspektrum (Gerät Varian 311A) bzgl. ${ }^{92}$ Mo (aus Aceton bzw. Toluol).

Das Filtrat wird konzentriert und an $\mathrm{SiO}_{2}$ (Säule $22 \times 3.5 \mathrm{~cm}$, Kieselgel 60 der Firma Merck, Zeitdauer ca. 8 h) chromatographiert. Mit Toluol/Et ${ }_{2} \mathrm{O}(10 / 1)$ lassen sich zunächst eine braungrüne und eine orange Zone eluieren. Durch wiederholte Chromatographie der braungrünen Zone unter den obigen Bedingungen, aber mit Toluol/ $\mathrm{Et}_{2} \mathrm{O}(100 / 1)$ als Eluens, lassen sich nacheinander eine braune (IV), grüne (III) und wieder braune (V) Zone isolieren. Die zweite, orange Zone wird ebenso behandelt, wobei mit Toluol $/ \mathrm{Et}_{2} \mathrm{O}(100 / 1)$ zunächst sehr schwach gefärbte Zonen ausgewaschen werden, bis VI mit Toluol/ $\mathrm{Et}_{2} \mathrm{O}(10 / 1)$ als orange Zone eluiert werden kann.

\section{Reaktionen von I bzw. II mit $(\mathrm{CO})_{5} \mathrm{Cr}(\mathrm{THF})$}

$335 \mathrm{mg}(0.50 \mathrm{mMol})$ II werden in $10 \mathrm{ml} \mathrm{CH}_{2} \mathrm{Cl}_{2}$ gelöst, mit einer Lösung von $0.50 \mathrm{mMol}(\mathrm{CO})_{5} \mathrm{Cr}$ (THF) in $200 \mathrm{ml}$ THF vereinigt und $17 \mathrm{~h}$ bei Raumtemperatur gerïhrt. Nach Ahziehen des Iösungsmittels wird der Rückstand in $10 \mathrm{ml} \mathrm{CH}_{2} \mathrm{Cl}_{2}$ aufgenommen und an $\mathrm{Al}_{2} \mathrm{O}_{3}$ (Akt. II/III) mit Toluol/Aceton (100/1) chromatographiert. Dabei eluiert man zuerst eine grünbraune Zone, sodann eine blaue Zone, die laut spektroskopischem Befund I (ca. $50 \mathrm{mg}$ ) enthält. Die Reinigung der ersten Zone erfolgt durch Chromatographie an $\mathrm{SiO}_{2}$ mit Toluol/Pentan $(1 / 1)$. VIII wird als rotbraune Zone in $20 \%$ Ausbeute isoliert, vorlaufende schwach gefärbte Zonen werden verworfen. Die Umkristallisation erfolgt aus Toluol/Pentan (1/1).

Die Umsetzung von I mit (CO) ${ }_{5} \mathrm{Cr}$ (THF) erfolgt nach dem gleichen Schema, bei der abschliessenden Chromatographie mit Toluol/Pentan (1/1) wird VII als erste, braune Zone eluiert, gefolgt von rotbraunem VIII. Die Ausbeuten sind in Tab. 3 enthalten. 
Darstellung von $\mathrm{Cp}_{2}^{\star} \mathrm{MO}_{2} \mathrm{Cr}_{2}\left(\mathrm{CO}_{5} \mathrm{OS}_{3}(\mathrm{IX})\right.$

Die Lösung von $90 \mathrm{mg}(0.35 \mathrm{mmol})\left(\mathrm{CH}_{3} \mathrm{CN}\right)_{3} \mathrm{Cr}(\mathrm{CO}, 2[22] \text { in } 30 \mathrm{ml} \mathrm{CH})_{3} \mathrm{CN}$ wird bei Raumtemperatur zur" hellgrünen Lösung von 160 ng 10.28 mmol) $\mathrm{Cp}_{2}^{\star} \mathrm{Mo}_{2} \mathrm{OS}_{3}$ in $30 \mathrm{ml}$ Toluol zugetropft. Nach $0.5 \mathrm{~h}$ Reaktionsdauer entzieht man der orangebraunen Mischung das Lösungsmittel. lost das Rohprodukt in $4 \mathrm{ml}$ Toluol und chromatographert an $\mathrm{SiO}_{2}$ (Säule $10 \times 2 \mathrm{~cm}$ ). IX wird mit Tolud als braungrüne Zone eluiert. Unkristallisation erfolgt aus Toluol Pentan $(5 / 1)$.

Darstellung con $\mathrm{Cp}_{2}^{\star} \mathrm{Mo}, \mathrm{Cr}, \mathrm{CO}, \mathrm{O}, \mathrm{S}, \mathrm{X}$

Zur orangen Lösung von $557 \mathrm{mg}(1.0 \mathrm{mmol}) \mathrm{Cp}_{2}^{\star} \mathrm{MO}_{2} \mathrm{O}_{2} \mathrm{~S}_{2}$ in $70 \mathrm{~m}$ Toluol wird eine Lösung aus $280 \mathrm{mg}$ (108 mmol) $\left(\mathrm{CH}_{3} \mathrm{CN}\right)_{3} \mathrm{Cr}(\mathrm{CO})_{3}[22]$ in $70 \mathrm{ml} \mathrm{CH}_{3} \mathrm{CN}$ getropft und anschliessend noch 3 h bei Raumtemperatur gerïhrt. Nach dem Entfernen des Losungsmitel lost man das schwarbraune Rohproduk in $10 \mathrm{ml}$ Toluol $/ \mathrm{CH}_{2} \mathrm{Cl}$,-Gemisch (1/1) und chromatographert an Sio, (Saule: $40 \times 3 \mathrm{~m}$ ). Mit Toluol eluiert man eine dunkelbraune breite Zone. dic zur weiteren Reinigung einer nochmaligen Chromatographie an SiO/ Toluol unterworfen wird. Die Umkristallisation aus Toluol hei $-25^{\circ} \mathrm{C}$ ergibt dunkelbrane verwachsene Balkchen.

\section{Literatur}

I J. Wachter, J. Coord. Chem. B. IS (1987) 219.

2 M. Herberhold. W. Krennitz, U. Thewalt. A. Razavi und H. Sobilhom. Angew Chem. $97(1985)$ 603: Angew. Chem. Int. Ed. Engl. 24(1985)601

3 M. Cousins und M.L.H Green. I Chem. Soc 1964, 1567: C. Condwell and K. Prout Acta Crystallogr.. B34 (1978) $93 \%$.

4 H. Arzoumanian, A. Baldy, M. Picrot und J.F. Petrignani, J. Organomet. (honı. 294 (1985) 327.

5 W.A. Hermann. R. Serrano und H. Bock. Angew. Chem. 96 (1984) 364: Anger. Chem. Int Fd Engl., 23 (1984) 383; W.A. Hermann. R. Serrano, U Küsthardt. M L. Zieghto I. Guggolz und T

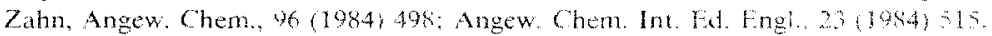

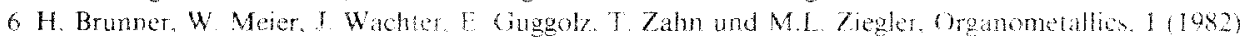
1107.

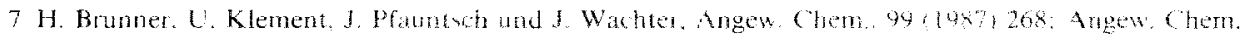
Ini. Ed. Engl.. 26 (1987) 230

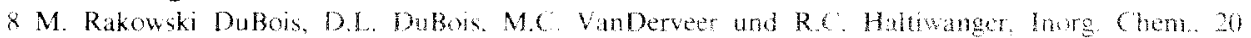
(1981) 3064

9 C.G. Young. M. Minelli, J.H. Enemark. G. Miessler. Y. Janelz. H. Katurmann und I Wachter Polyhedron. 5 (1986) 407

10 M. Minelij, J.H. Enemark. K. Wieghardt und M. Hahn. Fnorg. Chem. 22 148313952

11 K. Wieghardt, M. Gutmann. P. Chaudhuri. W. Gebert. M Minell, C.G. Young und 3 .H Enemark, Inorg. Chem., 24 (1985) 3151; S.F. Gheller. T.W. Hambley, R.T Brownes M.T. OConor. M.R. Snow und A.G. Wedd. I. Am. Chen. Soc. $105(1983) 1527$.

12 D.L. DuBois, W.K. Miller und M Rakowski DuBois, J. Am. Ghem. Soc. 103 (1981, 3424.

13 T. Chandler, D.L. Lichtenberger and I.H. Enemark, Inorg. Chem. 20 (1981:75.

14 I.M. Newsam und T.R. Habert. Thorg. Chem., 24 (1985) 491 und alort he lit.

Is D.L. Stevenson und L.F. Dahl, An. Chem. Soc, 89 (1967) 3721: W. Winer, W.P. Fehlhammer. A.T. Litw. G. Thiel und W. Bew. Ctem. Ber., 115 (1982) 1682

16. B. Nuber und M.L. Ziegler, unveroffentlisht.

17 H. Brunner, N. Janietz. I. Wachter, T Zahn und M.L. Ziegler. Angew. Chem. 97 (1985) 22: Angew Chem. Int. Fd. Engl. $24(1985)$ 133.

18 M.D. Curtis und P.D. Williams. Inorg. (hem., $19(1983) 2661$

19 O. lutz. A. Nolle und P. Kronck, Z.. Naturorsch. A. 32 (1977) 505: S.F. Gheller, P.A. Gazzana. A.F. Masters. R.T Brownlee M I Connos, A.G Wedd, J.R. Rodgers wnd M.R. Snow Inore. Chim. Acta. $54(1981) 1.131$.

20) Eine Zusammenfassung des derzeitigen Kenntnisstandes findet wh he M Herbothold wnd $B$ Schmidkon. J. Organomet. Chem. $308(1986) 35$

21 G.A. Zank, C.A. Jones. T.B. Ranchass und A.L. Rheingold, Inorg. Chem., 25 (1986) 1886.

22 W.P. Fehthammer, W.A. Hermann and K. Ofele in G. Brauer H Hrsg Handhub ate Präparativen Anorganischen Chenie, Enke Vohag. Stutgart. 1981. S. 2020 